\title{
Public-private partnerships improve health outcomes in individuals with early stage Alzheimer's disease
}

This article was published in the following Dove Press journal:

Clinical Interventions in Aging

10 April 2014

Number of times this article has been viewed

\author{
James E Galvin' \\ Magdalena I Tolea' \\ Nika George ${ }^{2}$ \\ Cheryl Wingbermuehle ${ }^{3}$
}

'Alzheimer Disease Center, Departments of Neurology, Psychiatry and Population Health, New York University Langone Medical Center, New York, NY, USA; ${ }^{2}$ Clinical Psychology Program, University of Missouri - St Louis, ${ }^{3}$ Alzheimer's Association, St Louis Chapter, St Louis, MO, USA
Correspondence: James E Galvin New York University Langone Medical Center, I 45 East 32nd Street, 2nd Floor, New York, NY 10016, USA

Tel + I 2 I 22630770

Fax + I 2122633273

Email james.galvin@nyumc.org
Purpose: In a collaborative effort between the Missouri Department of Health, Area Agencies on Aging (AAA), Alzheimer Association, and academic researchers, we tested whether early dementia detection and comprehensive care consultations would improve health outcomes in care receivers (CRs) and their family caregivers (FCGs), therefore addressing an important public health concern.

Participants and methods: A total of 244 community-dwelling older adults screened for early-stage dementia by the AAA field staff were referred to the Alzheimer Association and participated in Project Learn MORE (Missouri Outreach and Referral Expanded) (PLM) a 2-year, nonrandomized multisite intervention consisting of comprehensive care consultations to improve coping skills. PLM participants were compared against 96 controls receiving the Alzheimer Association's “usual services” between January 2011 and December 2012. We examined CR and FCG outcomes, including burden, care confidence, and mood, as effects of PLM, on delaying transitions in level of care.

Results: CRs showed improved knowledge $(P=0.002)$ and reduced depression $(P=0.007)$, while FCGs demonstrated improved knowledge $(P=0.003)$ and ability to identify sources of support for the $\mathrm{CR}(P=0.032)$ and for themselves $(P=0.043)$. However, FCGs were more burdened after PLM $(P=0.02)$, due to increased awareness of Alzheimer's disease. PLM delayed transitions in care (odds ratio [OR] 3.32, 95\% confidence level [CI]: 1.25-8.83) with the number needed to treat $=6.82$.

Conclusion: PLM was successful in improving detection of incident cases of dementia in the community and in connecting patients and their families with needed services. Our findings support the use of state agencies and community service partners to detect dementia. Early implementation of psychosocial interventions could have significant impact in improving patient- and family-centered outcomes, potentially providing a cost-efficient alternative to pharmacotherapy.

Keywords: dementia screening, AD8, social support, caregiver burden, transition in care

\section{Introduction}

Population aging will lead to a sharp increase in Alzheimer's disease and related dementias (collectively referred to as "AD" in this paper) in the next decades. In Missouri, this number is expected to exceed 130,000 by 2025 , with over 202,000 Missourians currently providing 2.3 million hours of unpaid care to patients who have dementia, valued at more than 2.5 billion dollars. ${ }^{1}$ About $40 \%$ of Missourians with AD are in the earlier stages of the disease and would therefore benefit from a variety of interventions - both pharmacologic and psychosocial - to improve patient- and family-centered outcomes.

There is lack of recognition of dementia in the community, with $50 \%$ of affected individuals being undiagnosed, particularly at the early stages. ${ }^{2}$ Many dementia 
screening tools do not fit with contemporary knowledge of early-stage AD (defined here as "mild-to-moderate dementia"), with "normal" range performance scores failing to detect impaired individuals. ${ }^{3}$ Promoting a mechanism to identify individuals with emerging cognitive impairment could connect families with appropriate resources and delay adverse outcomes. Dementia increases the risk of nursing home (NH) placement among the elderly by more than fivefold. ${ }^{4}$ However, Mittelman et al showed that enhanced family caregiver (FCG) support can delay placement by 1.5 years, ${ }^{5}$ suggesting that interventions offering a multidimensional approach are likely to be successful. ${ }^{6}$

The early stage in the disease process represents a critical time, when both FCGs and care receivers (CRs) can come to terms with the diagnosis and make future plans. ${ }^{\text {? }}$ Interventions at this time are feasible and have the potential to offer benefits to both CRs and FCGs regarding increased disease knowledge and confidence about dealing with the disease and its consequences, while potentially decreasing burden and providing resources. ${ }^{8}$ Early involvement of CRs in decision-making leads to positive outcomes in quality of life, enhances autonomy, and promotes individual preferences. ${ }^{9}$

New York University researchers, the Missouri Department of Health and Senior Services, ten Area Agencies on Aging (AAA), and four local Alzheimer's Association Chapters have collaborated in two projects aimed at increasing dementia detection and referral to services, to address this unmet need. The first was Project LEARN (Listen, Educate, Adjust, Resolve, Navigate). In this 12-month pilot study, staff in a designated AAA were trained to perform dementia screening using the AD8 (a brief interview to detect dementia) ${ }^{10,11}$ and referred individuals with early-stage dementia and their FCGs to local Chapters for supportive services and an intervention to increase coping skills. As a pilot study, no outcome measures were collected.

Project Learn MORE (Missouri Outreach and Referral Expanded) (PLM), which constitutes the focus of this report, was a 2-year study (January 2011-December 2012) that applied validated dementia screening methods to identify early stage $\mathrm{AD}$ in the community and connected affected individuals to needed services, while collecting outcomes to assess its impact on the health of both CRs and FCGs.

\section{Material and methods Project Learn MORE protocol}

As part of the study, staff members from four Missouri Chapters and ten AAAs were offered two all-day seminars discussing signs and symptoms of $\mathrm{AD}$, the administration and scoring of the AD8, ${ }^{10,11}$ the protocol for referring clients, the planned PLM intervention, and the program evaluation process. In-home assessments of older adults and referral of new clients determined to be cognitively impaired to the participating Alzheimer Association Chapters were conducted by the AAAs.

The AAA referrals were initiated by social workers, physicians, or by direct telephone calls from older adults needing support. Upon referral, the AAA Care Coordinators went into the home to conduct an assessment, to determine subjects' eligibility to receive services funded by the Older American's Act, including but not limited to nutrition and dining programs, care transitions, case-management services, FCG programs, transportation services, legal services, counseling, and adult day care. The assessment included the AD8 as a dementia screen. The AD8 score was entered in the AAA database along with all of the other assessment information. If the client scored 2 points or higher on the AD8, the care coordinator would suggest to the client (or their family member) that a referral be made to the Alzheimer Association. If the client did not want a referral, the AAA Coordinator would enter the reason given for declining the referral. If the client did want a referral to the chapter, this would be entered into the database. The AAA made the referral to the Alzheimer Association Chapters by (1) documentation through the statewide database (which was accessed by the PLM coordinators), (2) fax, (3) e-mail, or (4) phone call. In addition, AAA staff completed a 14-item yes-or-no answer survey to evaluate their experience administering the AD8.

The Alzheimer Association PLM Coordinators logged into the AAA database approximately twice per week to identify new referrals, then called the CR and FCG to gather information about their situation, ask exclusionary questions, explain PLM services, and to offer to schedule a care consultation. If a client was interested in scheduling a care consultation, an entry was made in the AAA database, showing the referral was made from the AAA to the chapter. The Alzheimer's Association Chapters offered comprehensive care consultations to the referred individuals and conducted pre- and posttest evaluations of the intervention. The services rendered by the AAA and Alzheimer Association constituted the standard of care practices for each agency. Deidentified data sets containing outcomes were analyzed with the approval of New York University's Institutional Review Board. 


\section{The AD8}

The AD8 dementia screening tool was used to identify older Missourians with dementia. ${ }^{10,11}$ The AD8 identifies problems in memory, problem-solving, orientation, and daily activities due to cognitive changes. It consists of eight yes/no items that give a total score ranging from $0-8$, where scores $>2$ suggest dementia. The AD8 is highly correlated with the Clinical Dementia Rating, ${ }^{10}$ neuropsychological testing, ${ }^{11}$ imaging and cerebrospinal fluid biomarkers. ${ }^{12}$ The AD8 can be completed by either the FCG, if available, or the CR. ${ }^{13}$ In our study, the AD8 was administered to all new clients of the ten participating AAAs. Per protocol, clients scoring $\geq 2$ were to be referred to the local Alzheimer Association Chapter for an individualized PLM care consultation.

\section{Project Learn MORE care consultation}

The PLM care consultation was an individualized 2-hour long consultation with the $\mathrm{CR}$ and FCG, conducted in a convenient location (eg, the CR home, the Chapter office, or via telephone). Given that the focus of PLM was on early stage dementia, AAA referrals were further evaluated by the Alzheimer Association Chapters to ensure that only early stage cases were included. PLM exclusion was based on having two or more of the following criteria: (1) consistent trouble recognizing close family members or friends; (2) change in ability to carry on a fairly normal conversation; (3) incontinence that is not attributed to another medical condition; and (4) requiring significant assistance with basic activities of daily living (eg, dressing or showering).

The PLM care consultation included a comprehensive assessment and individualized action plan. The former focused on both the CR and FCG and addressed physical, mental, social, and financial aspects of living with AD. Once specific needs were identified, an individualized action plan was created, including the identification of short-term goals to address client needs regarding coping skills, education, supportive services, and planning for the future, and referrals to services (eg, legal advice, driving assessments, etc). PLM participants could also apply for financial assistance of up to $\$ 1,000$ to cover expenses (eg, attorney fees, transportation costs, and dementia-related medications), removing barriers to access. Approximately $40 \%$ of PLM participants received grants.

Bimonthly follow up was conducted by phone, by the care consultant who conducted the care consultation or a graduate student or trained volunteer under the supervision of the care consultant. PLM follow up was continued until (1) CRs and FCGs accomplished the majority of goals; (2) the situation had stabilized; and/or (3) the PLM funding period ended. During the follow-up period, adjustments were made in the action plan to serve CR and FCG needs.

\section{Pre- and posttest evaluations}

Pre-posttest surveys were distributed to the family before the intervention and when they were discharged from the program. The FCGs were assessed using the 12-item Zarit Burden Inventory ${ }^{14,15}$ and the Dementia Care Confidence Scale. ${ }^{16}$ The CRs were also assessed for these domains, using modified questions addressing their concerns about the diagnosis. Individual burden and confidence items were measured using five-point Likert scales, where higher scores indicated higher levels of burden, confidence, and stress. Total burden and confidence were calculated by summing scores for individual items within the scales. Ten-point Likert scales were used to assess satisfaction with PLM, its impact on mood, coping skills, confidence, and "aging in place", and improved access to services for those families who received financial grants.

\section{Assessing impact of Project Learn MORE on transitions in care}

In addition, to assess the impact of the PLM intervention on transitions in level of care (assisted living [AL] or NH), during the PLM follow-up period, PLM clients $(n=244)$ were contacted and asked to provide information on the CR's living arrangements at the end of the PLM funding. As this was a community-based service, there was no placebo group for comparison. Instead, a "usual care" control group was created, consisting of early-stage CRs and FCGs $(n=96)$ who received other usual services offered by the Alzheimer Association Chapters (eg, Help line, non-PLM care consultations, etc), but not the PLM care consultation, during the same periods as PLM. The control group was tracked when they received these services, and their outcomes were recorded (death, placement, receipt of respite benefits, etc.). Non-PLM families eligible for the Alzheimer Association Respite program were sent a Respite Assistance Program application. They could apply to receive up to $\$ 500 /$ year (for a maximum of 2 years) to either (1) reimburse the primary caregiver for respite services to give the FCG time off or (2) to pay for products such as incontinence supplies, nutritional supplements, safety products, etc. Non-PLM clients were also eligible to receive a scholarship toward the cost of enrollment in the MedicAlert + Safe Return ${ }^{\circledR}$ program (MedicAlert Foundation, Turlock, CA, USA).

\section{Statistical analyses}

Analyses were conducted using SAS ${ }^{\circledR} 9.3$ (SAS Institute Inc., Cary, NC, USA). Descriptive statistics were used to 
present the data from the AAA survey on the experience of administering the AD8. Pre-post intervention change in disease burden, confidence, and other relevant factors were analyzed with the nonparametric Friedman test, for individual scale items, and with paired $t$-test, for total scale scores. The Friedman test was also used to compare FCG and CR satisfaction with PLM, and the impact of PLM on mood, confidence, and coping skills. The moderating effects of age, sex, race, chapter, and relationship of FCG to CR were tested with generalized linear models adapted for use with ordinal data, by testing the effect of an interaction term between these factors and a variable measuring pre-post status. Given that five tests were run for each outcome, a Bonferroni correction was applied to the set of analyses leading to a $P<0.01$ $(0.05 / 5=0.01$ and $0.05 / 4=0.013)$ being used to test for statistical significance.

To investigate the effect of PLM on delay in placement, we compared PLM participants with the "usual service" controls using logistic regression. Delay in transitions in care was defined as the $\mathrm{CR}$ residing in their private residence at the end of PLM as opposed to a move to either AL or $\mathrm{NH}$ during the same period. To further quantify the intervention effects, we estimated the relative ${ }^{17}$ and absolute ${ }^{18}$ risk reduction provided by PLM as well as the number needed to treat $(\mathrm{NNT}) .{ }^{19}$

\section{Results}

During the 2-year study, the AAAs conducted 3,682 assessments, of which 3,654 provided analyzable data (Figure 1). Using an AD8 cutoff point of $\geq 2,1,040$ (28\%) clients screened positive for cognitive impairment and thus were eligible for PLM. Of these, 313 clients were referred to the Chapters (30\% of those eligible), along with an additional four of the noneligible clients (with AD8 scores of $<2$ ), for a total of 317 referred clients. Of these, 244 (77\%) PLM dyads had a care consultation. Referred clients had a mean AD8 of 5.3 \pm 1.9 , suggesting mild-to-moderate dementia. AAA staff reported that the AD8 was easy to administer $(100 \%)$, useful to detect dementia (63\%), and helped them to discuss memory loss with clients $(76 \%)$.

The CRs were $61 \%$ female and $83 \%$ White, and $93 \%$ were $>60$ years old. Following PLM, the CRs were more confident in their AD knowledge $(P=0.002)$ and less likely to experience negative mood $(P=0.007)$. The CRs' perceived disease burden, concerns about ADLs, or driving were not changed following PLM (Table 1).

The FCGs were $67 \%$ females and $83 \%$ White, and $64 \%$ were $>60$ years old. Postintervention (Table 2 ), the FCGs were more confident in their knowledge $(P=0.003)$, about finding resources to help care for their affected family members $(P=0.032)$, and about their own health $(P=0.043)$.

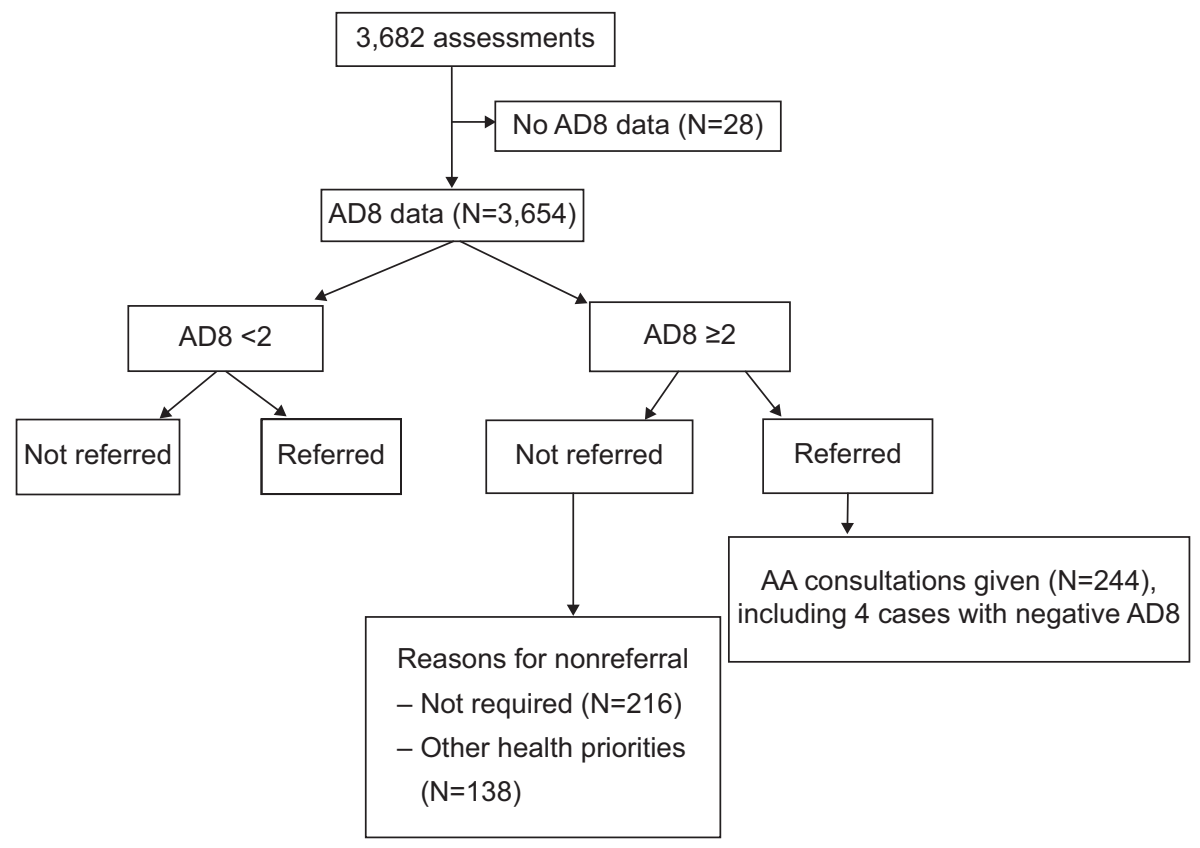

Figure I Flow of AAA clients in the PLM intervention.

Notes: The figure describes the initial number of client assessments conducted by AAA staff and the total number included in the analysis. Of the 3,654 assessments with valid AD8 data, 2,6I4 were cognitively intact and I,040 had AD8 scores indicative of cognitive impairment. Of these, 313 were referred to the Alzheimer Association for PLM services; 244 care consultations were given. Reasons for nonreferral are detailed.

Abbreviations: AA, Alzheimer's Association; AAA, Area Agencies on Aging (Missouri Department of Health); AD, Alzheimer's disease; AD8, eight-item dementia interview; PLM, Project Learn MORE (Missouri Outreach and Referral Expanded). 
Table I Mean differences in pre- and posttest in care receivers in Project Learn MORE (Missouri Outreach and Referral Expanded)

\begin{tabular}{|c|c|c|c|}
\hline & $\begin{array}{l}\text { Preintervention } \\
(\mathrm{N}=149)\end{array}$ & $\begin{array}{l}\text { Postintervention } \\
(\mathrm{N}=90)\end{array}$ & $\begin{array}{l}\text { Pre-post difference } \\
(\mathrm{N}=68)\end{array}$ \\
\hline & Mean (SD) & Mean (SD) & $P$-value \\
\hline \multicolumn{4}{|l|}{ Burden } \\
\hline BI (Family under stress) & $\mathrm{I} .87(\mathrm{I} . \mathrm{II})$ & $\mathrm{I} .78(\mathrm{I} . \mathrm{II})$ & 0.655 \\
\hline B2 (Family relations strained) & $\mathrm{I} .1 \mathrm{I}(\mathrm{I} .2 \mathrm{I})$ & $\mathrm{I} .2 \mathrm{I}(\mathrm{I} .08)$ & 0.547 \\
\hline B3 (Social life suffered) & $\mathrm{I} .48(\mathrm{I} .4 \mathrm{I})$ & $1.70(1.17)$ & 0.070 \\
\hline B4 (Lost control of life) & I.5I (1.34) & $\mathrm{I} .57(\mathrm{I} .36)$ & 0.879 \\
\hline B5 (Uncertain about future) & $\mathrm{I} .88(\mathrm{I} .4 \mathrm{I})$ & $\mathrm{I} .84(\mathrm{I} .2 \mathrm{I})$ & 1.000 \\
\hline Total burden score & $7.57(5.14)$ & $7.96(4.76)$ & 0.725 \\
\hline \multicolumn{4}{|l|}{ Confidence } \\
\hline $\mathrm{Cl}$ (Comfortable discussing AD) & $2.02(1.42)$ & $2.23(1.37)$ & 0.394 \\
\hline C2 (Confident in AD knowledge) & $1.66(1.18)$ & $2.16(1.18)$ & 0.002 \\
\hline C3 (Confident about resources) & $2.47(1.21)$ & $2.5 I(1.21)$ & 1.000 \\
\hline C4 (Confident to ask for help) & $2.99(1.18)$ & $2.85(1.10)$ & 0.456 \\
\hline Total confidence score & $8.85(3.62)$ & $9.32(3.91)$ & 0.746 \\
\hline \multicolumn{4}{|l|}{ Other items } \\
\hline DI (Worried about driving ability) & $1.06(1.30)$ & $\mathrm{I} .34(\mathrm{I} .4 \mathrm{I})$ & 0.862 \\
\hline AI (Worried about ADL abilities) & $1.35(1.21)$ & $1.53(1.20)$ & 0.537 \\
\hline MI (Feeling sad, blue, depressed, or hopeless) & $1.66(1.21)$ & $\mathrm{I} .43(1.03)$ & 0.007 \\
\hline
\end{tabular}

Notes: Pre-post differences were tested with nonparametric Friedman test and with paired $t$-test for differences in total scores in 68 care receivers with data on both pre- and postintervention outcomes.

Abbreviations: AD, Alzheimer's disease; ADL, activities of daily living; SD, standard deviation.

Table 2 Mean differences in pre- and posttest in family caregivers in Project Learn MORE (Missouri Outreach and Referral Expanded)

\begin{tabular}{|c|c|c|c|}
\hline & $\begin{array}{l}\text { Preintervention } \\
(\mathrm{N}=149)\end{array}$ & $\begin{array}{l}\text { Postintervention } \\
(\mathrm{N}=90)\end{array}$ & $\begin{array}{l}\text { Pre-post difference } \\
(\mathrm{N}=68)\end{array}$ \\
\hline & Mean (SD) & Mean (SD) & $P$-value \\
\hline \multicolumn{4}{|l|}{ Burden } \\
\hline BI (Not enough time for self) & $\mathrm{I} .56(\mathrm{I} . \mathrm{II})$ & $1.79(1.09)$ & 0.039 \\
\hline B2 (Stress juggling responsibilities) & $1.77(1.18)$ & $1.93(1.04)$ & 0.096 \\
\hline B3 (Angry when around CR) & $1.18(1.02)$ & $1.25(0.87)$ & 0.160 \\
\hline B4 (Other relationships affected) & $0.90(1.08)$ & $1.06(1.01)$ & 0.043 \\
\hline B5 (Strained when around CR) & $1.20(1.05)$ & $1.34(0.98)$ & 0.216 \\
\hline B6 (Health suffered) & $0.80(1.05)$ & $1.04(1.02)$ & $<0.001$ \\
\hline B7 (Privacy affected) & $0.86(1.04)$ & $1.20(1.16)$ & 0.008 \\
\hline B8 (Social life suffered) & $1.13(1.16)$ & $1.40(1.15)$ & 0.074 \\
\hline B9 (Lost control of life) & $\mathrm{I} .04(\mathrm{I} . \mathrm{II})$ & $1.32(1.11)$ & 0.021 \\
\hline BIO (Uncertain what to do about CR) & $2.08(\mathrm{I} .1 \mathrm{I})$ & $1.77(1.04)$ & 0.336 \\
\hline BII (Should do more about CR) & $2.05(\mathrm{I} . \mathrm{II})$ & $1.98(1.09)$ & 0.181 \\
\hline BI2 (Could care better for CR) & $1.89(1.05)$ & $1.84(1.07)$ & 1.000 \\
\hline Total burden score & $16.11(9.10)$ & $17.79(8.5 \mathrm{I})$ & 0.023 \\
\hline \multicolumn{4}{|l|}{ Confidence } \\
\hline $\mathrm{Cl}$ (Comfortable discussing AD) & $2.42(1.28)$ & $2.65(1.21)$ & 0.258 \\
\hline C2 (Confident in knowledge about AD) & $1.90(1.14)$ & $2.40(1.02)$ & 0.003 \\
\hline C3 (Confident about finding resources) & $2.46(1.07)$ & $2.85(0.98)$ & 0.032 \\
\hline C4 (Confident about coping strategies) & $2.22(\mathrm{I} . \mathrm{II})$ & $2.30(1.00)$ & 0.102 \\
\hline C5 (Confident identifying sources of support for own health) & $2.48(1.16)$ & $2.59(1.10)$ & 0.043 \\
\hline Total confidence score & $11.19(4.11)$ & $12.54(4.08)$ & 0.052 \\
\hline \multicolumn{4}{|l|}{ Other items } \\
\hline DI (Worried about CR's driving ability) & $\mathrm{I} .23(\mathrm{I} .4 \mathrm{I})$ & $\mathrm{I} . \mathrm{II}(\mathrm{I} .4 \mathrm{I})$ & 0.366 \\
\hline AI (Worried about CR's abilities with ADL) & $2.21(1.13)$ & $2.06(1.07)$ & 0.891 \\
\hline MI (Feeling sad, blue, depressed, or hopeless) & $1.38(1.14)$ & $1.40(1.01)$ & 0.033 \\
\hline
\end{tabular}

Note: Pre-post differences were tested with nonparametric Friedman test and with paired $t$-test for differences in total scores.

Abbreviations: AD, Alzheimer's disease; ADL, activities of daily living; $C R$, care receiver; SD, standard deviation. 


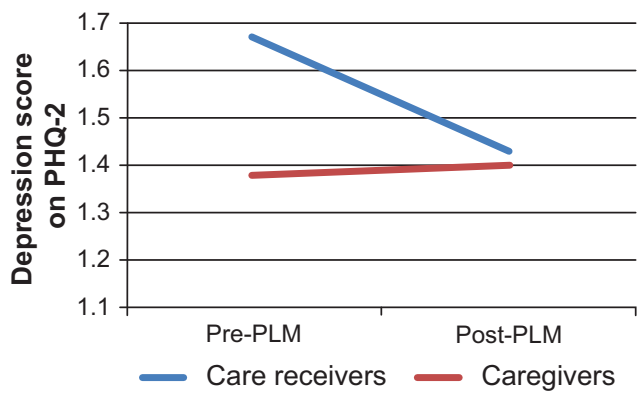

Figure 2 Change in mood score before and after the PLM intervention.

Notes: The figures present a comparison of pre- and postintervention change in mood score for CRs and their FCGs. The difference between CRs and FCGs in mood change was significant at $P<0.00 \mathrm{I}$.

Abbreviations: CR, care receiver; FCG, family caregiver; PLM, Project Learn MORE (Missouri Outreach and Referral Expanded); PHQ-2, Patient Health Questionnaire-2.

However, FCGs were more likely to feel depressed $(P=0.033)$ and burdened $(P=0.023)$. Compared with the CRs, FCGs were less likely to feel sad and depressed $(P=0.022)$ prior to PLM but reported similar mood scores after the intervention, suggesting the positive change in the CR mood was different from that in FCGs $(P<0.01)$ (Figure 2).

Postintervention, most participants reported that participation in PLM had reduced their fears about the new detection of $\mathrm{AD}$; that they appreciated the referral to community resources and physicians for formal diagnosis; and that treatment addressed their emotional and financial needs, increased their coping skills, and helped the CR remain at home longer than otherwise possible; these differences were greater in FCGs than in CRs (Table 3). After correcting for multiple comparisons, there were no differences related to PLM by sex age, race, FCG relationship, or Chapter.

One of the challenges facing PLM was noncompletion of the posttest evaluation. We tested whether any significant differences existed between the completers and noncompleters. The CR completers were similar to noncompleters in terms of age $(P=0.753)$, female sex $(P=0.550)$, race $(P=0.824)$, and AD8 score $(P=0.602)$. The FCG completers were more likely to be older $(P=0.016)$ and be spouses $(P=0.006)$ compared with noncompleters but were similar in terms of sex $(P=0.071)$, race $(P=0.274)$, and the AD8 score of their $\mathrm{CR}(P=0.470)$.

Compared with the "usual services" control group, referral to PLM delayed transitions to higher levels of care by threefold and offered significant risk reduction (Table 4). In terms of the NNT, PLM succeeded in delaying one transition from home to AL or NH for every seven participating CR-FCG dyads. PLM clients were similar to the "usual care" controls in terms of FCG age $(P=0.138)$ and sex $(P=0.208)$ but differed on FCG race/ethnicity $(83.1 \%$ of PLM FCGs were White and $13.8 \%$ Black, while among controls $54.7 \%$ were Black, and 39.0\% were Hispanics) $(P<0.001)$. PLM FCGs were more likely to be spouses than the "usual care" controls $(P<0.001)$. Other demographic information on CR was not available for the controls, and therefore, we were unable to compare the two groups on these factors. However, even after adjustment for FCG age, sex, race, and relationship to CR, PLM participants were more likely to avoid transitions in care (were still at home by the end of the study) compared with the controls (odds ratio $[\mathrm{OR}]=4.13,95 \%$ confidence interval $[\mathrm{CI}]$ : $1.18-14.44, P=0.026)$.

\section{Discussion}

The PLM program was designed with the expectations that (1) use of AD8 and the referral process would be readily adopted throughout the Missouri AAA system and would help identify community-dwelling older adults in early stages of dementia, and that (2) clients served would experience improved coping skills to deal with challenges of $\mathrm{AD}$, and improved awareness and usage of supportive services, and would report that the services offered and

Table 3 Post-care consultation survey in care receivers and family caregivers in PLM

\begin{tabular}{|c|c|c|c|}
\hline & Caregiver & Care receiver & $P$-value \\
\hline & Mean (SD) & Mean (SD) & \\
\hline SI (Satisfaction with PLM) & $9.02(1.65)$ & $8.42(2.25)$ & \\
\hline M2 (Reduced fears) & $8.39(1.86)$ & $7.44(2.37)$ & 0.001 \\
\hline M3 (Addressed emotional needs) & $8.46(1.83)$ & $7.47(2.34)$ & $<0.001$ \\
\hline C6 (Feel more comfortable discussing diagnosis with others) & $8.61(1.85)$ & $7.71(2.45)$ & $<0.001$ \\
\hline C7 (Increased coping skills) & $8.74(1.62)$ & $7.73(2.44)$ & 0.019 \\
\hline A2 (Will help CR remain at home longer than otherwise) & $8.28(2.31)$ & $8.09(2.17)$ & 0.001 \\
\hline S2 (Would recommend PLM) & $9.50(1.30)$ & $8.81(2.09)$ & 0.056 \\
\hline FI (Financial resources allowed access to services) & $9.24(1.72)$ & $9.20(1.83)$ & 0.001 \\
\hline F2 (Financial resources allowed more effective care of CR) & 9.11 (1.89) & $9.13(1.82)$ & 0.480 \\
\hline
\end{tabular}

Notes: Differences between caregivers and care receivers were tested with the nonparametric Friedman test.

Abbreviations: CR, care receiver; PLM, Project Learn MORE (Missouri Outreach and Referral Expanded); SD, standard deviation. 
Table 4 Effect of PLM on delay in transitions to care compared with control "usual services" provided by the Alzheimer's Association Chapter

\begin{tabular}{ll}
\hline Odds ratio $^{f}$ & 3.32 \\
& $(1.25,8.83)$ \\
Relative risk reduction (\%) & 64.10 \\
& $(14.96,84.84)$ \\
Absolute risk reduction (\%) & 14.67 \\
& $(3.70,25.64)$ \\
Number needed to treat & 6.82 \\
& $(3.90,27.03)$
\end{tabular}

Notes: A positive estimate indicates higher chances of delay in transitions associated with PLM; 'estimates obtained using logistic regression. Data are presented with estimates and the $95 \% \mathrm{Cl}$.

Abbreviations: $\mathrm{Cl}$, confidence interval; PLM, Project Learn MORE (Missouri Outreach and Referral Expanded).

knowledge gained would extend the time the CR remains at home.

AAA caseworkers reported high levels of satisfaction with the AD8 administration process and its effectiveness in identifying memory loss, suggesting that screening interventions may benefit from engaging the community partners providing support to CRs in early stages and their families. However, only one-third of clients who had AD8 scores indicative of impairment were actually referred for further services; this protocol highlights some of the challenges coordinating efforts when working with community service partners. The PLM experience replicated our experience with the Project LEARN pilot study, where 32\% were referred (data unpublished) but is higher than the proportion of referrals achieved in another similar intervention (where the referral rate by physicians was $17 \%) .{ }^{20}$ The lower referral rates in the latter may stem from physicians' lack of knowledge about Chapter services and/or reliance on a three-item word recall to trigger the referral rather than family input regarding cognitive and functional decline, and from limited time available for physician-patient interaction. In contrast, AAA staff may be more knowledgeable regarding locally available community resources and less pressed for time when assessing clients. Several reasons for nonreferral among impaired PLM clients were identified, including referral not required (Figure 1), which was surprising and indicates the need to ensure that dementia screening protocols are reinforced at the staff level to assure their correct implementation.

The receipt of PLM services resulted in a significant reduction in affective symptoms among the CRs, supporting reports of positive impact of FCG-CR interventions on depressive symptoms among patients. For example, lower depression has been reported among mild-to-moderate AD patients 6 months after receiving an individualized psychological support and pharmacological treatment intervention. ${ }^{21}$ The greater effect size in our study suggests that supportive interventions may fare at least as well as interventions that include pharmacologic components and may offer longer-lived benefits on CRs' mood. It may also be that the provision of supportive services to CRs may be more successful if (1) they are offered at earlier stages of impairment and (2) involve the FCG as well. PLM also increased CRs' confidence about AD knowledge by 30\%, suggesting that educational interventions that have increased knowledge and confidence among clinicians involved in dementia care ${ }^{22,23}$ may do the same for CRs.

Consistent with other similar reports, ${ }^{8,23}$ PLM increased FCGs' confidence about AD knowledge. Our findings suggest that given that many FCGs report feeling unprepared for their caregiver role and not knowing how to find and access available resources, ${ }^{24}$ by improving overall care confidence, programs to educate and link FCGs to community resources can improve long-term outcomes.

However, the FCGs in our study also experienced increased burden and depression. Our finding of increased depression in FCGs postintervention contradicts general reports of the small-to-moderate, but clinically significant, positive effects of psychosocial and educational interventions on FCG depression ${ }^{25}$ and mood. ${ }^{26}$ Regarding burden, the aspects that were most affected included lack of personal time; negative impact on relationships, health, and privacy; and lost life control. While these results contradict reports of the beneficial effects of psychosocial interventions on caregiver burden, ${ }^{27}$ the evidence for caregiver burden reduction is not as clear as that for depression. For example, in a trial of 81 mild-to-moderate dementia CR-FCG dyads randomly assigned to either an intervention to educate, support, and connect the family with existing resources, or to a control group (ie, waiting list), burden remained stable 6 months later. $^{28}$

There are several reasons for these discrepancies in results. First, it may be that the benefits on $\operatorname{mood} /$ stress are likely to occur among FCGs caring for loved ones in later dementia stages, when behavioral symptoms are increasingly problematic. Improving patient problem behaviors reduces FCG stress ${ }^{29}$ and aids in keeping the CR at home. ${ }^{30}$ In our study, the short duration between the AAA screening of incident cases, the PLM care consultation, and the understanding of AD impact on both CR and FCG may have contributed to the increase in burden and depression reported by the FCGs. Second, our measure of depression, derived from the Patient Health Questionnaire (PHQ)-2, ${ }^{31}$ differed from those used in other interventions. Third, positive effects on mood and burden may be limited to certain groups of FCGs with traits, 
such as low neuroticism ${ }^{32}$ or low baseline mastery, ${ }^{33}$ that were not captured in this study. The increased burden and depression in FCGs may be alleviated by longer durations of service provision than what was provided as part of PLM.

Our findings of delayed transitions in care are consistent with effects seen in other psychosocial support interventions, such as the New York University Caregiver Intervention (NYUCI). ${ }^{5,34}$ This longstanding project demonstrated a 577-day delay in nursing home placement compared with "usual care" controls. ${ }^{5}$ The shorter follow-up period may explain the higher rate of aging in place in the PLM group. Similarly, in a recent adaptation of the NYUCI to adult child FCGs (NYUCI-AC), the receipt of social support helped delay transitions to residential care $(\mathrm{AL} / \mathrm{NH})$ compared with controls. ${ }^{34}$ In our study, the impact of the intervention on placement did not differ by FCG relationship with the CR.

The PLM intervention shows similar positive long-term effects on FCGs to those seen in the COPE trial. In that trial, improvement in many areas, including care management and the ability to keep the patient at home, was observed 9 months after the CR-FCG intervention. ${ }^{35}$ These findings suggest that nonpharmacological interventions that focus on identifying and addressing the needs of dementia patients and their families are highly valued and of benefit to all those involved.

There were limitations to our study. First, the limitation associated with the PLM program is the absence of formal diagnoses to confirm the screening findings. While most CRs were referred to physicians for formal diagnosis by the Alzheimer Association Chapters, we have no data on how many followed through with this recommendation or the outcome of the diagnostic visits in those who did. The AD8 is a validated dementia screening test, comparable with gold standard diagnostic evaluations, and when used correctly, is accurate in detecting early-stage AD ( sensitivity $>84 \%$; specificity $>80 \%$; positive predictive value $>85 \%$; and negative predictive value $>70 \%$ ). The AD8 also works equally well for other forms of dementia. ${ }^{11}$ Our sample's mean AD8 score (5.3) suggests the PLM participants were at the mild-to-moderate stage of dementia. While we were unable to determine the exact cause of cognitive impairment, this does not lessen the benefits the CRs and FCGs received from the intervention and may more accurately reflect the general diagnosis of "dementia" in the community than the specific etiologies typically obtained at expert centers. Second, the AAA did not refer all eligible clients, leading to deviations from the intended protocol. This highlights the challenges in coordinating services between community service organizations and the need to better standardize workflow protocols. Third, inconsistencies between AAA and Chapter data made it difficult to directly test relationships between individuals seen by AAA and the outcomes of the PLM intervention. Only 40 surveys were completed by AAA staff, and the administration of pre- and posttests to CRs and FCGs by Chapters was inconsistent. Future studies involving multiple participating agencies may benefit from data that is collected in a standardized and consistent fashion, to reduce bias and threats to validity, while improving the generalizability of findings. Fourth, health outcomes were collected in a time interval relatively close to PLM intervention completion. A longer follow-up period is needed to determine the longterm benefits of the intervention. Lastly, our case-control analyses were based on data provided by one participating Chapter, which provided over $40 \%$ of the total PLM clients. Given that no differences by Chapter were observed, we feel confident that the control data were representative of the entire population of Chapter clients who received "usual services" during the implementation of the PLM interventions.

\section{Conclusion}

The PLM program should be considered a broad success, achieving its goals of identifying community-dwelling older adults with cognitive impairment and providing them with tools to increase their ability to cope with the disease. Our study clearly demonstrates the practical utility of public-private partnerships to improve service delivery and quality of life of families struggling with dementia. Multiagency programs focused on identifying, assessing, and addressing current and future care needs of cognitively impaired seniors and caregivers can have direct benefits for both CRs and FCGs. Identification of affected individuals as early in the disease process as possible coupled with the expertise that comes from repeated implementation of supportive service programs can help maximize these benefits, as observed in our study. New cases of dementia can be readily detected in the community, and community-based interventions, such as care consultations offered by the Alzheimer's Association, could have a significant impact in reducing resource utilization, delaying $\mathrm{NH}$ placement and potentially decreasing Medicare- and Medicaid-related costs, while improving patient- and caregiver-centered outcomes.

\section{Author contributions}

All authors had full access to all the de-identified data in the study. 
JE Galvin and C Wingbermuehle were responsible for the study concept and design, and supervised the study. N George and $\mathrm{C}$ Wingbermuehle participated in the acquisition of data. JE Galvin and MI Tolea performed the statistical analysis, interpreted the data, and drafted the manuscript. JE Galvin, MI Tolea, N George, and C Wingbermuehle critically reviewed the manuscript for important intellectual content. All authors provided administrative, technical, or material support. $\mathrm{C}$ Wingbermuehle obtained funding.

\section{Acknowledgments}

The authors thank the dedicated staff of the ten Missouri Area Agency on Aging offices and the four Missouri Alzheimer Association Chapters for their participation and assistance in completing this study. The authors specifically wish to acknowledge Glenda Meachum-Cain (Department of Health and Senior Services); Joetta Coen and Janie Bonham, RN (Mid-Missouri Chapter); Karen Clond, LMSW and Michelle Niedens, LSCSW (Heart of America Chapter); Angel King and Marci McKie (Southwest Missouri Chapter); and Debra Bryer, RN, Victoria Wingbermuehle, and David Hancock (St Louis Chapter).

\section{Disclosure}

JE Galvin holds the copyright for the AD8.

This project was supported by an Administration on Aging Alzheimer's Disease Demonstration Grant to the Missouri Department of Health and Senior Services (grant number: HHS-2010-AoA-A1-1012). JE Galvin and MI Tolea were supported by grants from the National Institutes of Health (grant numbers: P30 AG008051 and R01 AG040211) and a grant from the New York State Department of Health (grant number: DOH-2011-1004010353). Additionally, JE Galvin received grant support from the Michael J Fox Foundation, the Alzheimer Association, and the Morris and Alma Schapiro Fund. The funding agencies had no role in the design of the study; analysis or interpretation of the data; preparation of the manuscript; or decision to submit the manuscript for publication.

The authors report no other conflicts of interest in this work.

\section{References}

1. Alzheimer's Association. Alzheimer's Disease Facts and Figures 2010. Chicago, IL: Alzheimer's Association; 2010. Available from: http://www.alz.org/documents_custom/report_alzfactsfigures2010.pdf. Accessed July 1, 2013.

2. Galvin JE, Sadowsky CH; NINCDS-ADRDA. Practical guidelines for the recognition and diagnosis of dementia. J Am Board Fam Med. 2012;25(3):367-382.
3. Shiroky JS, Schipper HM, Bergman H, Chertkow H. Can you have dementia with an MMSE score of 30 ? Am J Alzheimers Dis Other Demen. 2007;22(5):406-415.

4. Eaker ED, Vierkant RA, Mickel SF. Predictors of nursing home admission and/or death in incident Alzheimer's disease and other dementia cases compared to controls: a population-based study. J Clin Epidemiol. 2002;55(5):462-468.

5. Mittelman MS, Haley WE, Clay OJ, Roth DL. Improving caregiver well-being delays nursing home placement of patients with Alzheimer disease. Neurology. 2006;67(9):1592-1599.

6. Montgomery R, Kwak J. TCARE: tailored caregiver assessment and referral. Am J Nurs. 2008;108(9 Suppl):S54-S57; quiz S57.

7. Whitlatch CJ, Judge K, Zarit SH, Femia E. Dyadic intervention for family caregivers and care receivers in early-stage dementia. Gerontologist. 2006;46(5):688-694.

8. Pinquart M, Sörensen S. Helping caregivers of persons with dementia: which interventions work and how large are their effects? Int Psychogeriatr. 2006;18(4):577-595.

9. Menne HL, Whitlatch CJ. Decision-making involvement of individuals with dementia. Gerontologist. 2007;47(6):810-819.

10. Galvin JE, Roe CM, Powlishta KK, et al. The AD8: a brief informant interview to detect dementia. Neurology. 2005;65(4):559-564.

11. Galvin JE, Roe CM, Xiong C, Morris JC. Validity and reliability of the AD8 informant interview in dementia. Neurology. 2006;67(11): 1942-1948.

12. Galvin JE, Fagan AM, Holtzman DM, Mintun MA, Morris JC. Relationship of dementia screening tests with biomarkers of Alzheimer's disease. Brain. 2010;133(11):3290-3300.

13. Galvin JE, Roe CM, Coats MA, Morris JC. Patient's rating of cognitive ability: using the AD8, a brief informant interview, as a self-rating tool to detect dementia. Arch Neurol. 2007;64(5):725-730.

14. Zarit SH, Reever KE, Bach-Peterson J. Relatives of the impaired elderly: correlates of feelings of burden. Gerontologist. 1980;20(6): 649-655.

15. Ballesteros J, Santos B, González-Fraile E, Muñoz-Hermoso P, Domínguez-Panchón AI, Martín-Carrasco M. Unidimensional 12-item Zarit Caregiver Burden Interview for the assessment of dementia caregivers' burden obtained by item response theory. Value Health. 2012; 15(8):1141-1147.

16. Connell CM, Boise L, Stuckey JC, Holmes SB, Hudson ML. Attitudes toward the diagnosis and disclosure of dementia among family caregivers and primary care physicians. Gerontologist. 2004;44(4): 500-507.

17. Barratt A, Wyer PC, Hatala R, et al; Evidence-Based Medicine Teaching Tips Working Group. Tips for learners of evidence-based medicine: 1. Relative risk reduction, absolute risk reduction and number needed to treat. CMAJ. 2004;171(4):353-358.

18. Laupacis A, Sackett DL, Roberts RS. An assessment of clinically useful measures of the consequences of treatment. N Engl J Med. 1988; 318(26):1728-1733.

19. Sinclair JC, Cook RJ, Guyatt GH, Pauker SG, Cook DJ. When should an effective treatment be used? Derivation of the threshold number needed to treat and the minimum event rate for treatment. J Clin Epidemiol. 2001;54(3):253-262.

20. Reuben DB, Roth CP, Frank JC, et al. Assessing care of vulnerable elders - Alzheimer's disease: a pilot study of a practice redesign intervention to improve the quality of dementia care. J Am Geriatr Soc. 2010;58(2):324-329.

21. Banerjee S, Willis R, Matthews D, Contell F, Chan J, Murray J. Improving the quality of care for mild to moderate dementia: an evaluation of the Croydon Memory Service Model. Int J Geriatr Psychiatry. 2007; 22(8):782-788.

22. Galvin JE, Meuser TM, Morris JC. Improving physician awareness of Alzheimer disease and enhancing recruitment: the Clinician Partners Program. Alzheimer Dis Assoc Disord. 2012;26(1):61-67.

23. Kuhn DR, Mendes de Leon CF. Evaluating an educational intervention with relatives of persons in the early stages of Alzheimer's Disease. Research on Social Work Practice. 2001;11(5):531-548. 
24. The National Center on Caregiving. Family Caregiving: State of the Art, Future Trends. San Francisco, CA: Family Caregiver Alliance; 2007. Available from: http://caregiver.org/sites/fca.huang.radicaldesigns.org/files/ pdfs/2007_asa_preconference_proceedings.pdf. Accessed March 14, 2014.

25. Schulz R, O’Brien A, Czaja S, et al. Dementia caregiver intervention research: in search of clinical significance. Gerontologist. 2002; 42(5):589-602.

26. Brodaty H, Green A, Koschera A. Meta-analysis of psychosocial interventions for caregivers of people with dementia. J Am Geriatr Soc. 2003;51(5):657-664.

27. Gitlin LN, Belle SH, Burgio LD, et al; REACH Investigators. Effect of multicomponent interventions on caregiver burden and depression: the REACH multisite initiative at 6-month follow-up. Psychol Aging. 2003;18(3):361-374.

28. Dias A, Dewey ME, D'Souza J, et al. The effectiveness of a home care program for supporting caregivers of persons with dementia in developing countries: a randomised controlled trial from Goa, India. PLoS One. 2008;3(6):e2333.

29. Bourgeois MS, Schulz R, Burgio LD, Beach S. Skills training for spouses of patients with Alzheimer's disease: Outcomes of an intervention study. Journal of Clinical Geropsychology. 2002;8(1):53-73.
30. Gitlin LN. Good news for dementia care: caregiver interventions reduce behavioral symptoms in people with dementia and family distress. Am J Psychiatry. 2012;169(9):894-897.

31. Kroenke K, Spitzer RL, Williams JB. The Patient Health Questionnaire-2: validity of a two-item depression screener. Med Care. 2003;41(11): 1284-1292.

32. Jang Y, Clay OJ, Roth DL, Haley WE, Mittelman MS. Neuroticism and longitudinal change in caregiver depression: impact of a spousecaregiver intervention program. Gerontologist. 2004;44(3):311-317.

33. Mahoney DF, Tarlow BJ, Jones RN. Effects of an automated telephone support system on caregiver burden and anxiety: findings from the REACH for TLC intervention study. Gerontologist. 2003;43(4):556-567.

34. Gaugler JE, Reese M, Mittelman MS. Effects of the NYU caregiver intervention-adult child on residential care placement. Gerontologist. 2013;53(6):985-997.

35. Gitlin LN, Winter L, Dennis MP, Hodgson N, Hauck WW. A biobehavioral home-based intervention and the well-being of patients with dementia and their caregivers: the COPE randomized trial. JAMA. 2010;304(9):983-991.
Clinical Interventions in Aging

\section{Publish your work in this journal}

Clinical Interventions in Aging is an international, peer-reviewed journal focusing on evidence-based reports on the value or lack thereof of treatments intended to prevent or delay the onset of maladaptive correlates of aging in human beings. This journal is indexed on PubMed Central, MedLine,

\section{Dovepress}

CAS, Scopus and the Elsevier Bibliographic databases. The manuscript management system is completely online and includes a very quick and fair peer-review system, which is all easy to use. Visit http://www.dovepress. com/testimonials.php to read real quotes from published authors. 